Functiones et Approximatio

49.2 (2013), 349-356

doi: $10.7169 / \mathrm{facm} / 2013.49 .2 .12$

\title{
TWINS OF POWERFUL NUMBERS
}

\author{
Valentin Blomer, Anita SchöBel
}

\begin{abstract}
For $k \geqslant 2$ we bound the number of pairs of consecutive $k$-full numbers.
Keywords: powerful numbers, additive problem, Thue equation, mixed-integer programming.
\end{abstract}

\section{Introduction}

A positive integer $n$ is called $k$-full for some integer $k \geqslant 2$ if $p \mid n$ implies $p^{k} \mid n$ for every prime $p$. This is a natural generalization of $k$-th powers, and it is easy to see that the sequence of $k$-full numbers is not much denser than the sequence of $k$-th powers: the number of $k$-full integers not exceeding $x$ is $\sim c_{k} x^{1 / k}$ for some constant $c_{k}>1$ as $x \rightarrow \infty$ (see e.g. [7, Section 14.4]). There are many interesting open questions associated with powerful numbers, and in particular additive problems often turn out to be hard.

In this note we want to consider a binary problem in $k$-full numbers, and for fixed $l \in \mathbb{Z} \backslash\{0\}$ estimate the number $\mathcal{N}_{k}(x ; l)$ of solutions to the equation $n-m=l$ with $k$-full numbers $n, m \leqslant x$. The trivial bound is $\mathcal{N}_{k}(x ; l) \ll x^{1 / k}$. The usual heuristic arguments based on density considerations predict

$$
\mathcal{N}_{2}(x ; l) \ll x^{\varepsilon}, \quad \mathcal{N}_{k}(x ; l) \ll 1, \quad k \geqslant 3 .
$$

Using the theory of Pell's equation one can show [8] that $\mathcal{N}_{2}(x ; l) \rightarrow \infty$ as $x \rightarrow \infty$, for any fixed $l \neq 0$. Proving (1.1) seems extraordinarily hard; it should be noted that even ternary additive problems in squarefull numbers are not well-understood (see [2]). The bounds in (1.1) follow essentially from the abc-conjecture: Chan has shown [3, Theorem 6] that the abc-conjecture implies $\mathcal{N}_{2}(x ; l) \ll_{l, \varepsilon} x^{\varepsilon}$ and hence a fortiori $\mathcal{N}_{k}(x ; l) \ll_{l, \varepsilon} x^{\varepsilon}$ for all $k \geqslant 2$. Recently (August 2012), Mochizuki has announced a proof of the $a b c$-conjecture. While this breakthrough result is still

\footnotetext{
The first author was supported by the Volkswagen Foundation and a Starting Grant of the European Research Council.
}

2010 Mathematics Subject Classification: primary: 11D45; secondary: 90C11 
under review, we investigate in this note how far one can get with comparatively elementary methods. We follow a recent paper of Chan who showed [3, Theorem 4]

$$
\mathcal{N}_{2}(x ; l) \ll_{l} x^{7 / 19} \log x
$$

where $7 / 19=0.368 \ldots$ For $k>2$, no non-trivial results have been obtained so far. We will improve (1.2) in a moment, but first we give a simple argument that provides non-trivial bounds for all $k \geqslant 2$.

Theorem 1. For $l \neq 0, k \geqslant 2$ and $\varepsilon>0$ one has $\mathcal{N}_{k}(x ; l) \ll_{\varepsilon, k} x^{\frac{2}{2 k+1}+\varepsilon}$. The implied constant is independent of $l$.

Proof. We can write each $k$-full number $n$ as $n=n_{1}^{k} n_{2}^{k+1} \cdots n_{k}^{2 k-1}$. This representation is unique if we require $n_{2}, \ldots, n_{k}$ to be squarefree and pairwise coprime, but even without this requirement a $k$-full number $n$ has, by a standard divisor estimate, at most $O\left(n^{\varepsilon}\right)$ representations of this form.

For two $k$-tuples $\mathbf{N}=\left(N_{1}, \ldots, N_{k}\right), \mathbf{M}=\left(M_{1}, \ldots, M_{k}\right)$ of positive real numbers let $\mathcal{N}_{k}(\mathbf{N}, \mathbf{M} ; l)$ be the number of solutions to the equation

$$
n_{1}^{k} \cdots n_{k}^{2 k-1}-m_{1}^{k} \cdots m_{k}^{2 k-1}=l
$$

where all variables are restricted to dyadic boxes $n_{j} \in\left[N_{j}, 2 N_{j}\right], m_{j} \in\left[M_{j}, 2 M_{j}\right]$. Let $\mathcal{N}_{k}^{*}(\mathbf{N}, \mathbf{M} ; l)$ denote the number of those $2 k$-tuples in $\mathcal{N}_{k}(\mathbf{N}, \mathbf{M} ; l)$ that satisfy in addition $\left(n_{j}, m_{j}\right)=1$ for $j=1, \ldots, k$. Then clearly

$$
\mathcal{N}_{k}(x ; l) \ll x^{\varepsilon} \max _{\substack{N_{1}^{k} \cdots N_{k}^{2 k-1} \leqslant x \\ M_{1}^{k} \cdots M_{k}^{2 k-1} \leqslant x}} \mathcal{N}_{k}(\mathbf{N}, \mathbf{M} ; l) \ll(x l)^{\varepsilon} \max _{\substack{N_{1}^{k} \cdots N_{k}^{2 k-1} \leqslant x \\ M_{1}^{k} \cdots M_{k}^{2 k-1} \leqslant x}} \max _{d \mid l} \mathcal{N}_{k}^{*}(\mathbf{N}, \mathbf{M} ; d) .
$$

Here and in the following, all estimates are uniform in $l$, and implied constants depend on $\varepsilon$ and $k$ at most. Obviously

$$
\mathcal{N}_{k}^{*}(\mathbf{N}, \mathbf{M} ; d) \ll \min \left(N_{1} \cdots N_{k}, M_{1} \cdots M_{k}\right)^{1+\varepsilon},
$$

since fixing $\left(n_{1}, \ldots, n_{k}\right)$ leaves $O\left(\left(m_{1} \cdots m_{k}\right)^{\varepsilon}\right)$ choices for $m_{1}, \ldots, m_{k}$, and the same argument holds with the roles of $n_{j}$ and $m_{j}$ reversed.

Alternatively, let us fix $n_{2}, \ldots, n_{k}, m_{2}, \ldots m_{k}$. In the case $k \geqslant 3$ we are left with a Thue equation in $n_{1}, m_{1}$, and by the main theorem in [1] the number of primitive (i.e. with $n_{1}$ and $m_{1}$ coprime) solutions is $O\left(l^{\varepsilon}\right)$, uniformly in the other variables. In the case $k=2$, we obtain a Pell-type equation $n_{1}^{2} n_{2}^{3}-m_{1}^{2} m_{2}^{3}=d$. Since $\left(n_{2}, m_{2}\right)=1$, the product $n_{2}^{3} m_{2}^{3}$ is a square if and only if $n_{2}$ and $m_{2}$ are squares in which case there are $O\left(d^{\varepsilon}\right)$ solutions. If $n_{2}^{3} m_{2}^{3}$ is not a square, then we bound the number of solutions by $O\left((x d)^{\varepsilon}\right)$, again uniformly in the other variables, since the fundamental unit of real quadratic fields is bounded below by an absolute constant, see e.g. [5, Hilfssatz 2] for a detailed proof. Hence we have the additional bound

$$
\mathcal{N}_{k}^{*}(\mathbf{N}, \mathbf{M} ; d) \ll(x d)^{\varepsilon} N_{2} \cdots N_{k} M_{2} \cdots M_{k} .
$$


Combining (1.3) - (1.5) we obtain

$$
\begin{aligned}
\mathcal{N}_{k}(x ; l) & \ll(x l)^{\varepsilon} \max _{\substack{N_{1}^{k} \cdots N_{k}^{2 k-1} \leqslant x \\
M_{1}^{k} \cdots M_{k}^{2 k-1} \leqslant x}} \min \left(N_{1} \cdots N_{k}, M_{1} \cdots M_{k}, N_{2} \cdots N_{k} M_{2} \cdots M_{k}\right) \\
& \leqslant(x l)^{\varepsilon} \max _{N^{k}, M^{k} \leqslant x} \min \left(N\left(\frac{x}{N^{k}}\right)^{\frac{1}{k+1}}, M\left(\frac{x}{M^{k}}\right)^{\frac{1}{k+1}},\left(\frac{x^{2}}{N^{k} M^{k}}\right)^{\frac{1}{k+1}}\right)
\end{aligned}
$$

since $N_{2} \cdots N_{k} \leqslant\left(x / N_{1}^{k}\right)^{1 /(k+1)}$ and similarly for $M_{2} \cdots M_{k}$. Using $\min (a, b, c) \leqslant$ $(a b)^{\frac{k}{2 k+1}} c^{\frac{1}{2 k+1}}$ we complete the proof of Theorem 1 .

The bounds are uniform in $l$ and the argument is not sensitive to signs (the number of solutions to $n_{1}^{2} n_{2}^{3}+m_{1}^{2} m_{2}^{3}=d$ is still $O\left(d^{\varepsilon}\right)$ ), hence the same argument shows:

Corollary 2. The number of representations of an integer $N$ as a sum of two $k$-full numbers is $O\left(N^{\frac{2}{2 k+1}+\varepsilon}\right)$.

This seems to be the first non-trivial result of this kind.

The rest of the paper is devoted to refinements of Theorem 1. Following [3], we change the argument leading to (1.5) by fixing only $2 k-3$ variables and considering the remaining count as a problem of bounding the number of rational points close to an (algebraic, but irrational) curve. To this end we use a result of Huxley which finally leads to an explicit, but non-trivial optimization problem. For $k=2$ this can be solved by hand, for $k>2$ we transform it into a linear mixed integer optimization problem. For $3 \leqslant k \leqslant 5$ we use FICO Xpress to find a numerical solution. This gives the following sharpening of Theorem 1 .

Theorem 3. For fixed $l \neq 0$ and $2 \leqslant k \leqslant 5$ we have $\mathcal{N}_{k}(x ; l) \ll x^{\gamma_{k}}$ for $\gamma_{2}>$ $61 / 180, \gamma_{3}=0.2665, \gamma_{4}=0.21, \gamma_{5}=0.174$.

Note that $61 / 180=0.338 \ldots$, so that Theorem 3 improves the main result of [3], cf. (1.2) above. As mentioned above, the bound for $k=2$ does not use a computer search. For $k>5$ the same method works, but the improvement compared to Theorem 1 becomes marginal. For relatively large values of $k$, recent work of Heath-Brown and Salberger on the Bombieri-Pila determinant method should be able to provide stronger improvements.

Remark. While the present article was in press, a paper by Chan [4] appeared in which the bound $\mathcal{N}_{3}(x ; l) \ll x^{45 / 139}(\log x)^{2}$ is proved. This is weaker than the corresponding bounds in Theorems 1 and 3 of the present paper. A recent preprint of Reuss [9] improves our value of $\gamma_{2}$ in Theorem 3 to $29 / 100$.

\section{Rational points close to a curve}

For positive real numbers $M, T, \Delta$ and $s \in \mathbb{N}$ define

$$
\begin{aligned}
H_{s}(M, T, \Delta):= & \left(M^{-s-1} T\right)^{\frac{1}{2 s+1}}+\left(\Delta^{\frac{1}{s}} M^{-s} T\right)^{\frac{1}{2 s+1}}+\Delta^{\frac{1}{2 s+1}} \\
& +\left(\Delta^{s^{2}+2 s-1} T^{s(s-1)}\right)^{\frac{1}{s(s+1)(2 s-1)}} .
\end{aligned}
$$


For positive real numbers $\lambda>0, M \geqslant 2, \alpha \notin \mathbb{Z}$ let $f:[0, M] \rightarrow \mathbb{R}$ be defined by

$$
f(x)=\lambda(1+x / M)^{\alpha} .
$$

We quote the following theorem of Huxley [6, Theorem 1] whose assumptions are easily verified for the present choice of $f$.

Proposition 1. Let $\lambda, M, \alpha$ and $f$ be as above. Fix $s \in \mathbb{N}$. Let $0<\Delta<1 / 2$ and $Q \geqslant 2+4 / \lambda^{1 / 2}$. Then the number of integer triples $(m, r, q)$ with $0 \leqslant m \leqslant M$, $1 \leqslant q \leqslant Q,(r, q)=1$ satisfying

$$
\left|f(m)-\frac{r}{q}\right| \leqslant \frac{\Delta}{Q^{2}}
$$

is at most

$$
\ll_{s, \alpha}(M(1+\lambda) Q)^{\varepsilon} \cdot M \cdot H_{s}\left(M, \lambda Q^{2}, \Delta\right) .
$$

Now let $\left(n_{1}, \ldots, n_{k}, m_{1}, \ldots, m_{k}\right)$ be a $2 k$-tuple counted by $\mathcal{N}^{*}(\mathbf{N}, \mathbf{M} ; d)$ and let us write

$$
X=N_{1}^{k} \cdots N_{k}^{2 k-1}
$$

for notational simplicity. We recall that $n_{j} \in\left[N_{j}, 2 N_{j}\right]$ and $m_{j} \in\left[M_{j}, 2 M_{j}\right]$. We can assume without loss of generality that $X$ is sufficiently large. The equation

$$
n_{1}^{k} \cdots n_{k}^{2 k-1}-m_{1}^{k} \cdots m_{k}^{2 k-1}=d
$$

implies $M_{1}^{k} \cdots M_{k}^{2 k-1} \asymp X$. Fix an index $j \in\{1, \ldots, k\}$. Then we conclude from (2.2) that

$$
\frac{n_{j}^{k+j-1}}{m_{j}^{k+j-1}}-\frac{m_{1}^{k} \cdots{\widehat{m_{j}}}^{k+j-1} \cdots m_{k}^{2 k-1}}{n_{1}^{k} \cdots{\widehat{n_{j}}}^{k+j-1} \cdots n_{k}^{2 k-1}}=\frac{d}{m_{j}^{k+j-1} n_{1}^{k} \cdots{\widehat{n_{j}}}^{k+j-1} \cdots n_{k}^{2 k-1}}
$$

where a hat denotes omission of the respective factor. Since

$$
|a-b| \leqslant\left|a^{k+j-1}-b^{k+j-1}\right| a^{2-j-k} \quad \text { for any } a, b>0,
$$

we obtain

$$
\left|\frac{n_{j}}{m_{j}}-\frac{\left(m_{1}^{k} \cdots{\widehat{m_{j}}}^{k+j-1} \cdots m_{k}^{2 k-1}\right)^{\frac{1}{k+j-1}}}{\left(n_{1}^{k} \cdots{\widehat{n_{j}}}^{k+j-1} \cdots n_{k}^{2 k-1}\right)^{\frac{1}{k+j-1}}}\right| \leqslant \frac{2 d}{M_{j}^{2}} \cdot \frac{N_{j} M_{j}}{X} .
$$

Now fix another index $i \neq j$ and write

$$
\frac{\left(m_{1}^{k} \cdots{\widehat{m_{j}}}^{k+j-1} \cdots m_{k}^{2 k-1}\right)^{\frac{1}{k+j-1}}}{\left(n_{1}^{k} \cdots{\widehat{n_{j}}}^{k+j-1} \cdots n_{k}^{2 k-1}\right)^{\frac{1}{k+j-1}}}=\lambda\left(1+\frac{\tilde{m}_{i}}{M_{i}}\right)^{\frac{k+i-1}{k+j-1}}
$$

where

$$
\tilde{m}_{i}=m_{i}-M_{i}, \quad \lambda=\left(\frac{M_{i}}{m_{i}}\right)^{\frac{k+i-1}{k+j-1}} \frac{\left(m_{1}^{k} \cdots{\widehat{m_{j}}}^{k+j-1} \cdots m_{k}^{2 k-1}\right)^{\frac{1}{k+j-1}}}{\left(n_{1}^{k} \cdots{\widehat{n_{j}}}^{k+j-1} \cdots n_{k}^{2 k-1}\right)^{\frac{1}{k+j-1}}} .
$$


Without loss of generality we can assume that $M_{i}$ is an integer. Then $\tilde{m}_{i} \in\left[0, M_{i}\right]$ is an integer, and we can count the number of triples $\left(n_{j}, m_{j}, \tilde{m}_{i}\right)$ satisfying $(2.3)$ using Proposition 1 with

$$
\alpha=\frac{k+i-1}{k+j-1} \notin \mathbb{Z}, \quad Q=2 M_{j}, \quad M=M_{i}, \quad \Delta \asymp \frac{N_{j} M_{j}}{X}, \quad \lambda \asymp \frac{N_{j}}{M_{j}}
$$

(where implicit constants may depend on $d$ and $k$ ). The assumptions $M \geqslant 2$, $Q \geqslant 2+4 / \lambda^{1 / 2}$ can be satisfied by multiplying $Q$ and/or $M$ with a fixed constant if necessary. If $X$ is sufficiently large, the condition $\Delta<1 / 2$ will be satisfied automatically, unless possibly $k=2$ and $N_{2} M_{2} \ll 1$, in which case Theorem 3 is trivial by (1.5). Proposition 1 now implies

$$
\mathcal{N}^{*}(\mathbf{N}, \mathbf{M} ; d) \ll_{\varepsilon, d, k, s} X^{\varepsilon} \frac{N_{1} \cdots N_{k} M_{1} \cdots M_{k}}{N_{j} M_{j}} H_{s}\left(M_{i}, N_{j} M_{j}, \frac{N_{j} M_{j}}{X}\right)
$$

for any choice of $i \neq j \in\{1, \ldots, k\}$ and any fixed $s \in \mathbb{N}$. By symmetry we can interchange the roles of $N$ and $M$ and obtain in the same way

$$
\mathcal{N}^{*}(\mathbf{N}, \mathbf{M} ; d) \ll_{\varepsilon, d, k, s} X^{\varepsilon} \frac{N_{1} \cdots N_{k} M_{1} \cdots M_{k}}{N_{j} M_{j}} H_{s}\left(N_{i}, N_{j} M_{j}, \frac{N_{j} M_{j}}{X}\right) .
$$

Combining $(1.3),(1.4),(2.4)$ and $(2.5)$, we obtain $\mathcal{N}_{k}(x ; l) \ll_{k, l, s_{0}, \varepsilon} x^{\varepsilon} \max _{X \leqslant x} \mathcal{M}_{k}(X)$ where

$$
\begin{aligned}
& \mathcal{M}_{k}(X)= \max _{\substack{N_{1}^{k} \cdots N_{k}^{2 k-1}=X \\
M_{1}^{k} \cdots M_{k}^{2 k-1}=X}} \min \left(N_{1} \cdots N_{k}, M_{1} \cdots M_{k}\right. \\
&\left.\min _{1 \leqslant i \neq j \leqslant k} \min _{s \leqslant s_{0}} \frac{N_{1} \cdots N_{k} M_{1} \cdots M_{k}}{N_{j} M_{j}} H_{s}\left(\max \left(N_{i}, M_{i}\right), N_{j} M_{j}, \frac{N_{j} M_{j}}{X}\right)\right)
\end{aligned}
$$

for any fixed $s_{0}$. Here we used that $H_{s}$ is decreasing in the first variable. We are now left with solving the minimax problem (2.6).

\section{A minimax problem with disjunctive constraints}

For $k=2$ it is easy to solve (2.6) by hand. We substitute $N_{2}=X^{1 / 3} N_{1}^{-2 / 3}$, $M_{2}=X^{1 / 3} M_{1}^{-2 / 3}$ and write for notational simplicity $N=N_{1}, M=M_{1}$. We consider only the case $s=4$ and obtain

$$
\begin{gathered}
\mathcal{M}_{2}(X) \leqslant \max _{N, M \leqslant X^{1 / 2}} \min \left((X N)^{1 / 3},(X M)^{1 / 3}, N M H_{4}\left(\max (N, M), \frac{X^{2 / 3}}{(N M)^{2 / 3}},\right.\right. \\
\left.\left.\frac{1}{X^{1 / 3}(N M)^{2 / 3}}\right), \frac{X^{2 / 3}}{(N M)^{2 / 3}} H_{4}\left(\frac{X^{1 / 3}}{\min \left(N^{2 / 3}, M^{2 / 3}\right)}, N M, \frac{N M}{X}\right)\right) .
\end{gathered}
$$


On any given hyperbola $N M=Y$, the expression $\min (\ldots)$ takes its maximum at $N=M=Y^{1 / 2}$. Hence

$$
\begin{aligned}
\mathcal{M}_{2}(X) \leqslant \max _{N \leqslant X^{1 / 2}} \min \left((X N)^{1 / 3}, N^{2} H_{4}\left(N, \frac{X^{2 / 3}}{N^{4 / 3}}, \frac{1}{X^{1 / 3} N^{4 / 3}}\right)\right. & \\
& \left.\frac{X^{2 / 3}}{N^{4 / 3}} H_{4}\left(\frac{X^{1 / 3}}{N^{2 / 3}}, N^{2}, \frac{N^{2}}{X}\right)\right) .
\end{aligned}
$$

A lengthy, but straightforward calculation shows that the expression $\min (\ldots)$ is given by

$$
\begin{cases}N^{2}, & N \leqslant X^{2 / 19} \\ X^{2 / 27} N^{35 / 27}, & X^{2 / 19} \leqslant N \leqslant X^{1 / 8} \\ X^{7 / 108} N^{37 / 27}, & X^{1 / 8} \leqslant N \leqslant X^{1 / 5} \\ X^{53 / 108} N^{-41 / 54}, & X^{1 / 5} \leqslant N \leqslant X^{19 / 62} \\ X^{2 / 3} N^{-4 / 3}, & X^{19 / 62} \leqslant N \leqslant N^{1 / 2}\end{cases}
$$

and hence $\mathcal{M}_{2}(X) \leqslant X^{61 / 180}$ as claimed.

For $k>2$ it becomes complicated to solve (2.6) by hand. In order to prepare for a computer search we linearize the problem by writing $N_{j}=X^{\nu_{j}}, M_{j}=X^{\mu_{j}}$. For notational simplicity we write $\nu:=\nu_{1}+\ldots+\nu_{k}, \mu:=\mu_{1}+\ldots+\mu_{k}$. This gives $\mathcal{M}_{k}(X) \ll X^{\beta}$ where

$$
\begin{gathered}
\beta=\max _{\begin{array}{c}
k \nu_{1}+\ldots+(2 k-1) \nu_{k}=1 \\
k \mu_{1}+\ldots+(2 k-1) \mu_{k}=1 \\
\nu_{j}, \mu_{j} \geqslant 0
\end{array}} \min \left(\nu, \mu, \min _{1 \leqslant i \neq j \leqslant k} \min _{s \leqslant s_{0}}\left(\nu+\mu-\nu_{j}-\mu_{j}\right.\right. \\
\left.\left.+\min \left(L_{i, j, s}(\boldsymbol{\nu}, \boldsymbol{\mu}), L_{i, j, s}(\boldsymbol{\mu}, \boldsymbol{\nu})\right)\right)\right)
\end{gathered}
$$

with

$$
\begin{aligned}
L_{i, j, s}(\boldsymbol{\nu}, \boldsymbol{\mu})=\max & \left(\frac{-(s+1) \nu_{i}+\nu_{j}+\mu_{j}}{2 s+1}, \frac{(s+1)\left(\nu_{j}+\mu_{j}\right)-s-s^{2} \nu_{i}}{s(2 s+1)},\right. \\
& \left.\frac{\nu_{j}+\mu_{j}-1}{2 s+1}, \frac{\left(s^{2}+2 s-1\right)\left(\nu_{j}+\mu_{j}-1\right)+s(s-1)\left(\nu_{j}+\mu_{j}\right)}{s(s+1)(2 s-1)}\right)
\end{aligned}
$$

by $(2.6)$ and $(2.1)$. Using $k \nu_{1}+\ldots+(2 k-1) \nu_{k}=k \mu_{1}+\ldots+(2 k-1) \mu_{k}=1$ we can eliminate the variables $\nu_{k}$ and $\mu_{k}$ and define the polyhedron

$$
\begin{array}{r}
D:=\left\{\left(\nu_{1}, \ldots, \nu_{k-1}, \mu_{1}, \ldots, \mu_{k-1}\right) \in[0,1]^{2 k-2} \mid k \nu_{1}+\ldots+(2 k-2) \nu_{k-1}\right. \\
\left.k \mu_{1}+\ldots+(2 k-2) \mu_{k-1} \leqslant 1\right\} .
\end{array}
$$

An inspection of (3.1) shows that we need to maximize a function of the type

$$
\min _{i \in I} \max _{j \in J} f_{i j}(\boldsymbol{\nu}, \boldsymbol{\mu}) \longrightarrow \max , \quad(\boldsymbol{\nu}, \boldsymbol{\mu}) \in D
$$


where $f_{i j}(\boldsymbol{\nu}, \boldsymbol{\mu})=\left(a_{i j}\right)^{t} \boldsymbol{\nu}+\left(b_{i j}\right)^{t} \boldsymbol{\mu}+c_{i j}$ are certain linear functions with matrices $\left(a_{i j}\right),\left(b_{i j}\right),\left(c_{i j}\right)$ with $1 \leqslant j \leqslant 4,1 \leqslant i \leqslant 2 k(k-1) s_{0}+2$ that are determined by (3.1). The nested occurrence of min and max requires some preparation. It is not hard to see that $0 \leqslant f_{i j}(\boldsymbol{\nu}, \boldsymbol{\mu}) \leqslant 1$ for $(\boldsymbol{\nu}, \boldsymbol{\mu}) \in D$ and each pair $(i, j)$. For each pair $(i, j)$ we introduce an integer (in fact boolean) variable $z_{i j} \in\{0,1\}$. Then (3.2) is equivalent to the following linear mixed-integer program

$$
\begin{aligned}
(\mathrm{MIP}) & \max f \\
\text { s.t. } f_{i j}(\boldsymbol{\nu}, \boldsymbol{\mu}) & =a_{i j} \boldsymbol{\nu}+b_{i j} \boldsymbol{\mu}+c_{i j} \text { for all } i \in I, j \in J \\
f_{i} & \geqslant f_{i j} \text { for all } i \in I, j \in J \\
f_{i} & \leqslant f_{i j}+M\left(1-z_{i j}\right) \text { for all } i \in I, j \in J \\
f & \geqslant f_{i} \text { for all } i \in I \\
\sum_{j \in J} z_{i j} & \geqslant 1 \text { for all } i \in I \\
(\boldsymbol{\nu}, \boldsymbol{\mu}) & \in D \\
z_{i j} & \in\{0,1\} \text { for all } i \in I, j \in J \\
f, f_{i}, f_{i j} & \text { free }
\end{aligned}
$$

if $M \geqslant f_{i j}(\nu, \mu)-f_{i^{\prime} j^{\prime}}(\nu, \mu)$ for all $i, i^{\prime} \in I, j, j^{\prime} \in J$ and for all $(\boldsymbol{\nu}, \boldsymbol{\mu}) \in D$. In our case we chose $M=1$ and $s_{0}=20$.

In order to solve the above mixed-integer program (MIP) we used FICO Xpress v7.2. Without any help, Xpress was not even able to find a feasible solution. From symmetry considerations and motivated by the case $k=2$, one may conjecture that the optimal solution should occur on the diagonal $\boldsymbol{\nu}=\boldsymbol{\mu}$. In order to generate a feasible solution we did a pre-run in which we added this additional symmetry condition. This type of variable fixing made the problem tractable and generated an optimal solution under the extra condition $\boldsymbol{\nu}=\boldsymbol{\mu}$. Using this solution as starting solution Xpress was then able to solve (MIP) and justified that the symmetry we required indeed holds.

We remark on the side that it would be interesting to find a proof for the general validity of this symmetry assumption. In this case the optimization problem (3.2) for $k=3$ can be solved by hand rather easily leading to the exponent $105 / 394 \approx$ $0.26649 \ldots$

\section{References}

[1] E. Bombieri, W. Schmidt, On Thue's equation, Invent. Math. 88 (1987), 69-81.

[2] T. Browning, K. v. Valckenborgh, Sums of three squareful numbers, Exp. Math. 21 (2012), 204-211.

[3] T.H. Chan, Twin squarefull numbers, J. Australian Math. Soc. 93 (2012), $43-51$.

[4] T.H. Chan, Twin cubefull numbers, Int. J. Number Theory 9 (2013), 17-26. 
[5] T. Estermann, Einige Sätze über quadratfreie Zahlen, Math Ann. 105 (1931), 653-662.

[6] M. Huxley, The rational points close to a curve $I V$, in: Proceedings of the Session in Analytic Number Theory and Diophantine Equations, Bonner Math. Schriften 360 (2003), 36pp.

[7] A. Ivić, The Riemann zeta-function, Wiley 1985.

[8] W.L. McDaniel, Representations of every integer as the difference of powerful numbers, Fibon. Quart. 20 (1982), 85-87.

[9] T. Reuss, Pairs of k-free numbers, consecutive square-full numbers, arxiv:1212.3150.

Addresses: Valentin Blomer: Mathematisches Institut, Bunsenstr. 3-5, 37073 Göttingen, Germany;

Anita Schöbel: Institut für Numerische und Angewandte Mathematik, Lotzestr. 16-18, 37083 Göttingen, Germany.

E-mail: blomer@uni-math.gwdg.de, schoebel@math.uni-goettingen.de

Received: 22 November 2012 